\title{
RECOPILACIÓN DE LA INFORMACIÓN BIOGEOGRÁFICA, ANÁLISIS DE PATRONES ECOLÓGICOS, CONSERVACIÓN Y MAPA POTENCIAL DE GRAELLSIA ISABELAE (GRAELLS, 1849) (LEPIDOPTERA, SATURNIIDAE) EN LA PROVINCIA DE CUENCA, ESPAÑA
}

\author{
J. I. de Arce Crespo*, S. Jiménez Mendoza* \& P. Sánchez Fernández
}

\begin{abstract}
RESUMEN
J. I. de Arce Crespo, S. Jiménez Mendoza \& P. Sánchez Fernández. 2010. Recopilación de la información biogeográfica, análisis de patrones ecológicos, conservación y mapa potencial de Grael/sia isabelae (Graells, 1849) (Lepidoptera, Saturniidae) en la provincia de Cuenca, España. Grael/sia, 66(1): 9-20.

Se ha realizado un estudio sobre la mariposa nocturna Graellsia isabelae (Graells, 1849) en la provincia de Cuenca, la cual se encuentra protegida en la región de Castilla-La Mancha. Se ha elaborado un mapa de distribución biogeográfica, ampliándose su presencia en un total de 8 cuadrículas UTM de 10×10 km. Además, se ha analizado la distribución de la especie con respecto a los espacios naturales protegidos de la provincia observando que está presente en la mayoría de los mismos. Así mismo, se ha realizado un cruce de datos de distribución en cuadrículas UTM 1x1 km con diferentes capas de sistemas de información geográfica (mapa forestal español, modelo digital del terreno, geología y caracterización climática de Allué-Andrade), obteniendo preferencias de patrones ecológicos, tales como la vegetación actual, caracterización climática, geología y distribución altitudinal. El hábitat principal de $G$. isabelae son zonas ocupadas por pino laricio (Pinus nigra subsp. salzmanii) y pino albar ( $P$. sylvestris). Por último, se ha realizado un mapa de distribución potencial según la vegetación, la caracterización climática y la altitud, observándose que la distribución de G. isabelae en la provincia de Cuenca puede ser considerablemente mayor que la conocida hasta la fecha.
\end{abstract}

Palabras clave: Lepidoptera; Saturniidae; Graellsia isabelae; Serranía de Cuenca; distribución geográfica; espacio natural protegido; sistemas de información geográfica; especie amenazada.

\begin{abstract}
J. I. de Arce Crespo, S. Jiménez Mendoza \& P. Sánchez Fernández. 2010. Compilation of biogeographical information, analysis of ecological patterns, conservation and potential map of Graellsia isabelae (Graells, 1849) (Lepidoptera, Saturniidae) in the province of Cuenca, Spain. Grael/sia, 66(1): 9-20 (in Spanish).

In the present paper, we update the distributional information of the endangered moth Graellsia isabelae (Graells, 1849) in Cuenca (Castilla-La Mancha) and provide relevant ecological information. The species is present in 14 localities corresponding to $1410 \times 10 \mathrm{~km}$ UTM squares; 8 of them are published for the first time in this study. We corroborate the presence of the species in all the Protected Areas in Cuenca. Using the known occurrence data and a set of digital environmental variables such as current vegetation, climate, geology and
\end{abstract}




\begin{abstract}
elevation, we found that the distribution of the species seems to be mainly determined by the presence of Scots pine ( $P$. sylvestris) and Austrian pine (Pinus nigra subsp. salzmanii) forests. Finally, a potential distribution map for the moth in Cuenca suggests that the species could inhabit other areas where it is presently unknown.
\end{abstract}

Key words: Graellsia isabelae; Saturniidae; Serranía de Cuenca; geographic distribution; protected natural area; geographic information systems; threatened species.

\section{Introducción}

La mariposa isabelina, Graellsia isabelae (Graells, 1849) (Lepidoptera: Saturniidae), es uno de los lepidópteros ibéricos más vistosos y admirados de la Península Ibérica desde su descubrimiento (Gómez-Bustillo \& Fernández-Rubio, 1976). Vive en la Península Ibérica principalmente en las sierras interiores de la parte oriental, encontrándose en diversas poblaciones dispersas y más o menos aisladas unas de otras: Sierra de Guadarrama; Sierra de Béjar; Sierra de Albarrracín, Montes Universales y Serranía de Cuenca; Sierras de Javalambre, Gúdar y Peñagolosa; Sierras de Cazorla, Segura y Alcaraz; Pirineos; y Puertos de Beceite (Gómez-Bustillo \& Fernández-Rubio, 1976; De Viedma \& GómezBustillo, 1985; Koschwitz et al., 1985; Masó \& Ylla, 1989; Blázquez et al., 2000; Calle et al., 2000; Galante \& Verdú, 2000; Pérez De Gregorio et al., 2002) y la última población conocida y más meridional de la Península en Sierra María en la provincia de Almería (Ibáñez Gázquez et al., 2008). Fuera de España, se encuentra en las montañas del Jura y los Alpes en Francia, alcanzando el cantón de Valais en Suiza (Masó \& Ylla, 1989; Guyot, 1998).

Graellsia isabelae es un lepidóptero de hábitos nocturnos y crepusculares que, en la Serranía de Cuenca, tiene una de sus localidades típicas de observación y recolección donde es "pieza segura" según Gómez-Bustillo \& Fernández-Rubio (1974). La Serranía de Cuenca, dentro del Sistema Ibérico meridional y junto a las provincias de Guadalajara, Teruel, Valencia y Castellón, alberga una de las mayores poblaciones de este lepidóptero en cuanto a extensión y abundancia (Masó \& Ylla, 1989; López Sebastián et al., 2001; García-Barros \& Herranz, 2001). G. isabelae, se alimenta principalmente de las especies Pinus nigra subsp. salzmanii (pino negral, laricio o salgareño) y Pinus sylvestris (pino albar) (Gómez-Bustillo \& Fernández-Rubio, 1976), aunque también se han citado otras plantas nutricias en cría en cautividad, tales como Pinus pinaster (pino rodeno), Pinus uncinata (pino negro) y Cedrus atlantica (cedro) (Masó \& Ylla, 1989).
Graellsia isabelae aparece citada con diversas figuras en una serie de listados de protección de especies: Convenio de Berna (Anexo III) como "especie protegida" (Viejo et al., 1991); en la UICN en 1996 la ha catalogado como DD "datos insuficientes"; en la Directiva Hábitats (Directiva 92/43/CEE) se cita en el Anexo II como "especie de interés comunitario para cuya conservación es necesario designar zonas especiales de conservación" y en el Anexo V: "especie de interés comunitario cuya recogida en la Naturaleza y cuya explotación pueden ser objeto de medidas de gestión"; en el Catálogo Nacional de Especies Amenazadas como especie de "interés especial" (Orden 10 de marzo de 2000); en el Anexo II y VI de la Ley 42/2007, de 13 de diciembre, del Patrimonio Natural y de la Biodiversidad; en la revisión del Libro Rojo de los lepidópteros ibéricos (De Viedma \& Bustillo, 1985) la han incluido como "endemismo" a nivel de subespecie; y en la zona de estudio se ha citado en el Anexo IV "de interés especial” según el Decreto 33/98 por el que se crea el Catálogo Regional de Especies Amenazadas de Castilla-La Mancha. Sin embargo, G. isabelae no está citada en la última versión del Libro Rojo de los Invertebrados de España (Verdú \& Galante, 2006) y diversos autores señalan que se encuentra "fuera de peligro", aunque podría tratarse como "vulnerable" debido a la regresión de su hábitat (Galante \& Verdú, 2000).

Así mismo, G. isabelae está citada como especie protegida mediante la categoría de "interés especial" dentro de las normas de declaración de los siguientes espacios naturales protegidos (en adelante ENP) de la provincia de Cuenca: Parque Natural de la Serranía de Cuenca (Decreto 99/2006, de 1 de agosto); Monumento Natural de la Hoz de Beteta y Sumidero Mata Asnos (Decreto 23/2004, de 2 de marzo); Monumento Natural de las Lagunas de Cañada del Hoyo (Decreto 18/2007, de 20 de marzo); Monumento Natural Muela Pinilla y del Puntal (Decreto 281/2003, de 23 de septiembre); Monumento Natural Serrezuela de Valsalobre (Decreto 76/2003, de 13 de mayo); y Reserva Natural 
Tabla 1.- Muestreos de Grael/sia isabeae realizados durante el año 2003 Se indica la localidad, fecha, UTM $10 x 10$ km y altitud.

Table 1.- Sampling of Grael/sia isabelae during 2003. Locality, date, $10 \times 10 \mathrm{~km}$ UTM square and altitude are indicated.

\begin{tabular}{lccc}
\hline MUESTREO & FECHA & UTM & ALTITUD \\
\hline Hoz Huécar y Palancares y Tierra Muerta & $29 / 03 / 2003$ & 30TWK73 y 30TWK83 & 1000 y 1130 \\
Palancares y Tierra Muerta & $04 / 04 / 2003$ & 30TWK83 & 1130 \\
Dehesa Cotillas (Buenache de la Sierra) & $05 / 04 / 2003$ & 30TWK84 & 1190 \\
Beamud & $12 / 04 / 2003$ & $30 T W K 94$ & 1320 \\
El Maillo- Las Majadas & $13 / 04 / 2003$ & $30 T W K 96$ & 1430 \\
Cañamares y Priego & $19 / 04 / 2003$ & 30TWK67 & 1050 \\
Fuente de Valdosillos (Huélamo) & $30 / 04 / 2003$ & 30TXK06 & 1440 \\
Palancares y Tierra Muerta & $03 / 05 / 2003$ & 30TWK83 & 1130 \\
El Maillo- Las Majadas & $10 / 05 / 2003$ & 30TWK96 & 1430 \\
La Mogorrita & $14 / 05 / 2003$ & 30TXK06 & 1600 \\
Laguna del Marquesado y Campillos Sierra & $15 / 05 / 2003$ & 30TXK15 y 30TXK14 & 1475 y 1300 \\
Carretera del Cañigral-Salvacañete & $24 / 05 / 2003$ & 30TXK34 & 1310 \\
Beteta y Carrascosa de la Sierra & $31 / 05 / 2003$ & 30TWK78 & 1100 \\
La Mogorrita & $06 / 06 / 2003$ & 30TXK06 & 1600 \\
Masegosa- Cruce Carretera a Peralejos de Las Truchas & $07 / 06 / 2003$ & 30TWK88 & 1560 \\
\hline
\end{tabular}

de la Laguna del Marquesado (Decreto 222/2004, de 1 de junio). También se encuentra citada en el Parque Natural del Alto Tajo (Decreto 204/1999, de 21 de septiembre) y en el Monumento Natural de Palancares y Tierra Muerta (Decreto 2/2001, de 16 de enero), pero en estos casos no se hace una mención explícita a su estado de protección.

Se han publicado varios trabajos sobre la distribución biogeográfica de $G$. isabelae a nivel peninsular sin información detallada de su localización en cuadrículas UTM (Gómez-Bustillo et al., 1974; De Viedma \& Gómez-Bustillo, 1976, 1985; GómezBustillo \& Fernández-Rubio, 1976; Masó et al., 1989; Galante \& Verdú, 2000; Pérez De Gregorio et al., 2002). No hay muchos estudios que contemplen información biogeográfica a nivel de UTM en la Península Ibérica, salvo algunos trabajos a nivel regional en Andalucía (Fernández Irurita, 2008) y en la Comunidad Valenciana (Montagud et al., 2001). Conocer la distribución de las mariposas protegidas es importante para su conservación (Chefaoui \& Lobo, 2007) por lo que el cartografiado de los recursos biológicos resulta imprescindible para la identificación de áreas de concentración de la diversidad biológica y la gestión de reservas naturales (García-Barros et al., 2004).

Los Sistemas de Información Geográfica (G.I.S.) pueden servir para identificar patrones ecológicos teóricos mediante la superposición de mapas temáticos sobre la distribución biogeográfica de una especie determinada en un territorio dado $y$, una vez identificados, se pueden utilizar para la confección de diferentes mapas de distribución potencial, tal y como se ha realizado con la especie objeto del presente estudio, G. isabelae (Chefaoui \& Lobo, 2007) y otros lepidópteros diurnos (Romo et al., 2006; Jiménez-Valverde et al., 2008), datos que pueden ser utilizados para tomar decisiones de conservación por los responsables de la administración en materia de Medio Ambiente.

En el presente estudio se informa sobre la distribución biogeográfica de $G$. isabelae en la provincia de Cuenca, se ha elaborado un mapa de distribución en cuadrículas UTM donde figura la presencia de la especie en los diferentes ENP de la provincia. También se identifican aquellas preferencias sobre ciertos patrones ecológicos y se elabora un mapa de distribución potencial con los datos obtenidos.

\section{Material y métodos}

Se han revisado diversas publicaciones nacionales e internacionales y se han visitado diversos lugares de la provincia de Cuenca indicados en la Tabla 1, realizándose durante el año 2003 un muestreo nocturno utilizando lámparas de mercurio. El resto de los años, desde 2004 hasta 2009, los ejem- 
Tabla 2.- Citas sin publicar de Grael/sia isabelae recopiladas en la provincia de Cuenca. Se indica la localidad, UTM 10x10 km, UTM $1 x 1$ km, altitud, fecha, colector y número de ejemplares y sexo (Colectores: ADR: A. Díaz-Romeral; FPA: Francisco Piñas Amor; J \& A: S. Jiménez Mendoza y J.I. de Arce Crespo; PSF: Pablo Sánchez Fernández; SRC: Sandra RamírezCárdenas Amer; MPP: Marcelino de los Mozos Pascual; y MLR: María López Rodríguez). Las localidades que vienen indicadas con asterisco amplían distribución.

Table 2.- New records of Graellsia isabelae in the province of Cuenca. Locality, $10 \times 10 \mathrm{~km}$ UTM square, $1 \times 1 \mathrm{~km}$ UTM square, altitude, date, collector and number of individuals and sex are indicated. (Collectors: ADR: A. Díaz-Romeral; FPA: Francisco Piñas Amor; J \& A: S. Jiménez Mendoza y J.I. de Arce Crespo; PSF: Pablo Sánchez Fernández; SRC: Sandra Ramírez-Cárdenas Amer; MPP: Marcelino de los Mozos Pascual; y MLR: María López Rodríguez). * Localities that expand the known distribution of the species in Cuenca.

\begin{tabular}{|c|c|c|c|c|c|c|}
\hline LOCALIDAD & UTM 10X10 & UTM 1 X1 & ALTITUD & FECHA & COLECTOR & EJEMPLARES \\
\hline K-27 Carretera Cuenca- Valencia* & 30SWK81 & 30SWK8314 & 1100 & 03/05/1978 & ADR leg. & 3 Machos \\
\hline 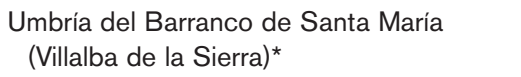 & 30TWK57 & 30TWK5973 & 1170 & $17 / 04 / 2009$ & SRC & 1 Macho \\
\hline Finca de Albaladejito* & 30TWK63 & 30TWK6836 & 920 & $22 / 04 / 2002$ & MMP & 1 Macho \\
\hline Cañamares* & 30TWK67 & \multicolumn{2}{|c|}{ Se desconoce Se desconoce } & $5-11 / 07 / 1974$ & FPA leg. & 1 Hembra (oruga) \\
\hline Mirabueno (Alrededores de Cuenca Capital) & 30TWK73 & 30TWK7336 & 1000 & $\begin{array}{l}11 / 05 / 1977 \\
05 / 05 / 1978 \\
27 / 05 / 1978\end{array}$ & $\begin{array}{l}\text { ADR leg. } \\
\text { ADR leg. } \\
\text { ADR leg. }\end{array}$ & $\begin{array}{l}1 \text { Macho } \\
1 \text { Macho } \\
1 \text { Macho }\end{array}$ \\
\hline Barrio del Castillo (Cuenca Capital) & 30TWK73 & 30TWK7336 & 1000 & $\begin{array}{l}17 / 04 / 2003 \\
01 / 05 / 2003\end{array}$ & $\begin{array}{l}J \& A \\
J \& A\end{array}$ & $\begin{array}{l}1 \text { Macho } \\
1 \text { Macho }\end{array}$ \\
\hline Dehesa Boyal (Fresneda de la Sierra) ${ }^{*}$ & 30TWK77 & 30TWK7071 & 1.100 & $28 / 05 / 2007$ & PSF & 1 Macho \\
\hline Hoz de Beteta (Monumento Natural)* & 30TWK78 & зоTWK7389 & 1100 & $31 / 05 / 2003$ & $J \& A$ & 1 Hembra \\
\hline Collado de la Nava (Valdecabras) & 30TWK84 & 30TWK8546 & 1.340 & $\begin{array}{l}09 / 06 / 2007 \\
23 / 05 / 2009\end{array}$ & $\begin{array}{l}\text { PSF } \\
\text { PSF }\end{array}$ & $\begin{array}{l}1 \text { Macho } \\
1 \text { Hembra }\end{array}$ \\
\hline Arroyo de la Madera (Uña) & 30TWK85 & 30TWK8555 & 1110 & $06 / 06 / 2004$ & $J \& A$ & 1 Hembra \\
\hline Sierra de los Barrancos (Monte de U.P.118) & ) $30 T W K 87$ & зоTWK8871 & 1130 & $21 / 04 / 2009$ & MLR & 1 Macho, 1 Hembra \\
\hline Rincón del Buitre- El Hosquillo & 30TWK96 & 30TWK9167 & 1190 & $14 / 04 / 2002$ & MMP & 1 Macho \\
\hline \multirow[t]{2}{*}{ La Mogorrita* } & 30TXK06 & ЗоTXK0466 & 1710 & $02 / 06 / 2000$ & MMP & 1 Macho \\
\hline & & & 1710 & $14 / 05 / 2003$ & $J \& A$ & 1 Macho \\
\hline Carretera al Cañigral (Salvacañete)* & 30TXK24 & Se desconoce & 1320 & $14 / 05 / 1979$ & ADR leg. & 1 Macho \\
\hline
\end{tabular}

plares de G. isabelae fueron observados a primeras horas del día, mientras se realizaban muestreos de mariposas diurnas. Además, se ha consultado la colección pública del Museo de las Ciencias de Castilla-La Mancha y la colección privada de Armando Díaz-Romeral.

Se ha realizado un cartografiado UTM 10x10 $\mathrm{km}$ de lado mediante el programa ArcView con toda la información recopilada de las citas propias, de la bibliografía (Aistleitner \& Aistleiner, 1998; Galante \& Verdú, 2000; Montagud et al., 2001; Piñas, 2003) y citas procedentes de comunicaciones personales. Se ha superpuesto la distribución biogeográfica obtenida con los ENP y futuros ENP de la provincia de Cuenca. Posteriormente, los datos disponibles se han cartografiado en cuadrículas
UTM de $1 \mathrm{x} 1 \mathrm{~km}$ y se han cruzado con la información de la cartografía temática GIS mediante el programa informático ArcView para identificar los diferentes patrones ecológicos de la especie. Por último, se ha realizado un mapa potencial de distribución geográfica UTM 1x1 y 10x10 km de lado de $G$. isabelae con las variables de vegetación, Modelo Digital del Terreno de las curvas de nivel y caracterización climática.

Se ha utilizado la información disponible de forma gratuita de Sistemas de Información Geográfica a través de Internet:

http://www.jccm.es/maydr/inap/forms2/inapf001.php\#capas: ENP y futuros ENP de la provincia de Cuenca. 


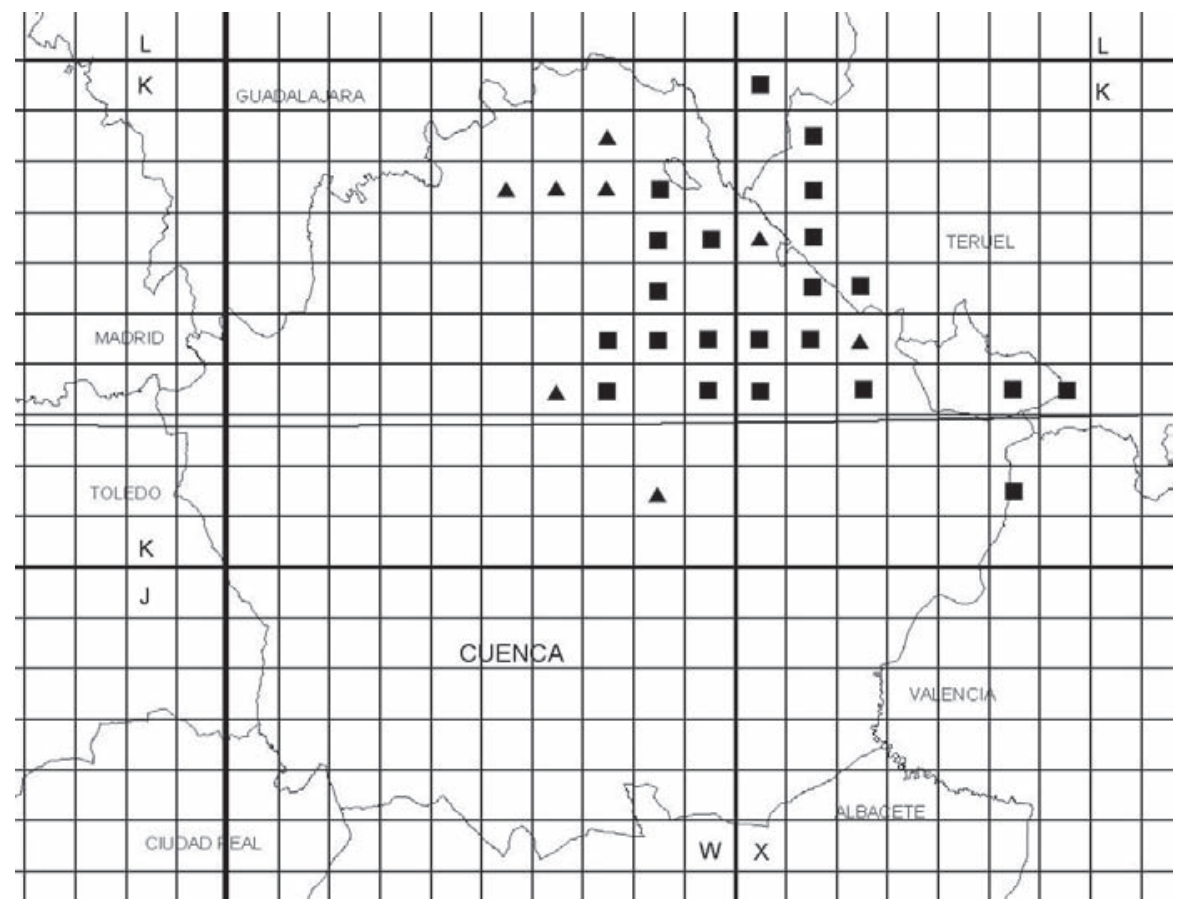

Fig. 1.- Mapa de distribución corológica de Graellsia isabelae en cuadrículas UTM de 10 km de lado en la provincia de Cuenca y provincias limítrofes. (Datos de la Tabla 1 y la bibliografía: Aistleitner \& Aistleitner, 1998; Galante \& Verdú, 2000; Montagud et al., 2001; Piñas, 2003; Joaquín Baixeras, com. pers.). Leyenda: el símbolo cuadrado indica las citas obtenidas de la bibliografía y el símbolo triángulo indica las cuadrículas que amplían la distribución en el presente estudio.

Fig. 1. - Distribution map of Grael/sia isabelae in 10x10 km UTM squares in Cuenca and neighbouring provinces using the records form Table 1 and the literature: Aistleitner \& Aistleitner, 1998; Galante \& Verdú, 2000; Montagud et al., 2001; Piñas, 2003; Joaquín Baixeras, com. pers). Squares: records from the literature, triangles: new records.

http://www.irnase.csic.es/users/microleis/mimam/seisnet.htm: Geología.

http://www.mma.es/portal/secciones/biodiversidad/banco dat os/info disponible/mapa subregiones fitoclim.htm: Caracterización climática de Āllué-Andrade (1990).

También se han empleado los recursos del Mapa Forestal de España (Ministerio de Medio Ambiente) a escala 1:50.000 y curvas de nivel de la provincia de Cuenca. La localización exacta de las localidades se ha realizado mediante un dispositivo GPS y para citas antiguas se ha utilizado el recurso de los mapas realizados por el Instituto Geográfico Nacional a escala 1:25.000 y 1: 50.000 de la provincia de Cuenca.

Para reconocer los ejemplares de Graellsia isabelae no ha sido necesaria la consulta de guías entomológicas especializadas, ya que es una mariposa fácil de identificar y que no ofrece ningún tipo de confusión.

\section{Resultados y Discusión}

\section{DISTRIBUCIÓN COROLÓGICA}

En la Tabla 2 aparece la información más reciente recopilada sobre Graellsia isabelae en la provincia de Cuenca, se indica la localidad, UTM 10x10, UTM 1x1, altitud, fecha, colector, número de ejemplares y sexo de los mismos. Se presentan un total de 14 localidades de las cuales 8 amplían la distribución conocida. En la Tabla 2 aparecen un total de 21 ejemplares, predominando los machos ya que únicamente 5 de ellos son hembras. El ejemplar de la localidad de Cañamares (30TWK67) es una oruga que fue recolectada por Francisco Piñas Amor entre los días del 5 al 11 de julio de 1974, la crió en cautividad y cuando emergió resultó ser una hembra, en su etiqueta no viene recogido cuando se produjo la emergencia a imago. 


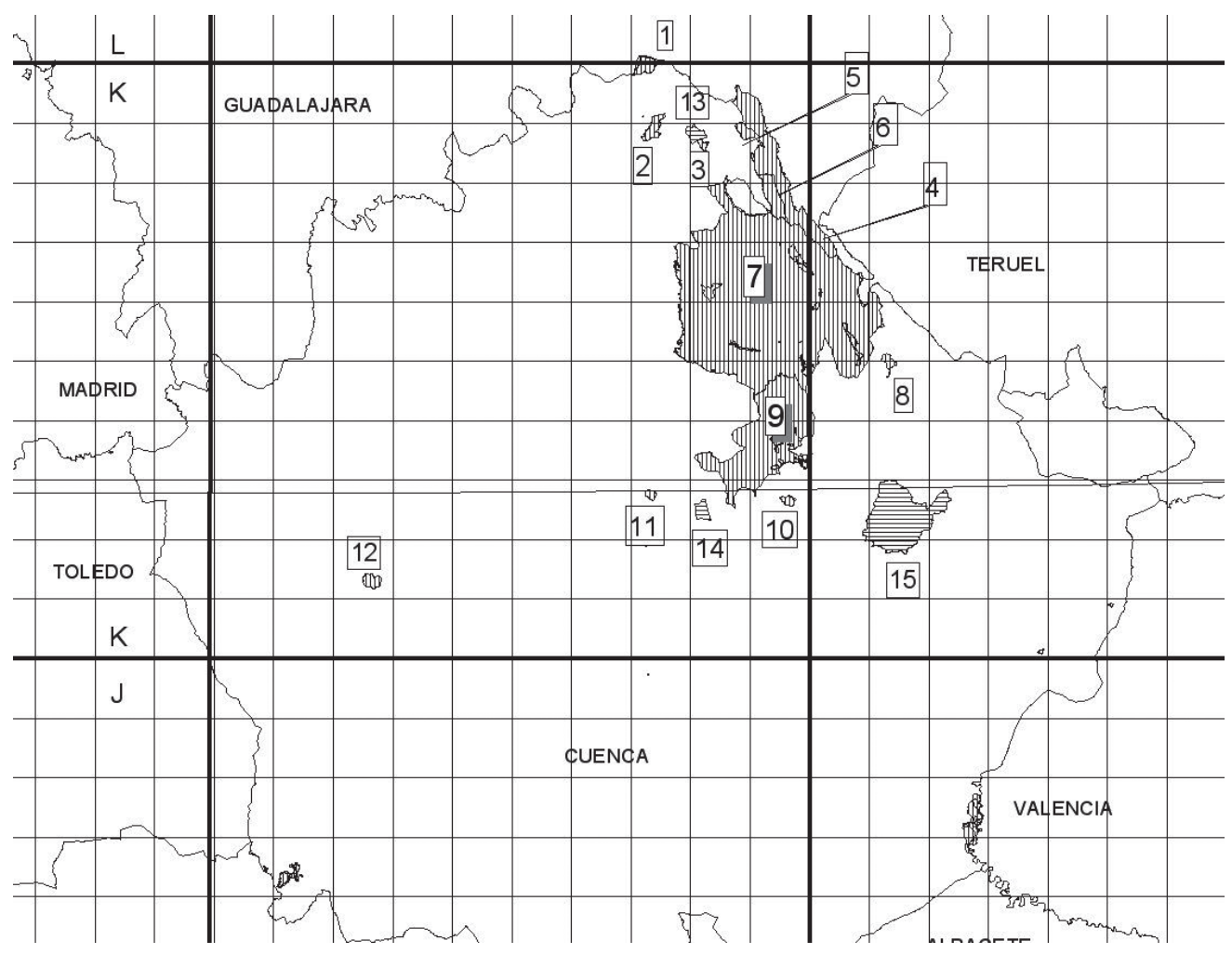

Fig. 2.- Localización de los diferentes Espacios Naturales Protegidos (ENP) (representado mediante líneas verticales) y futuros ENP (representado mediante líneas horizontales) de la Provincia de Cuenca con respecto a las coordenadas UTM de $10 \times 10$ km. Leyenda: 1 . Monumento Natural (M. N.) Serrezuela de Valsalobre; 2. M. N. Hoz de Beteta y Sumidero Mata Asnos; 3. M. N. Torcas de Lagunaseca; 4. Parque Natural (P.N.) del Alto Tajo; 5, M. N. Muela Pinilla y del Puntal; 6. M. N. Nacimiento del río Cuervo; 7. P.N. de la Serranía de Cuenca; 8. Reserva Natural (R. N.) de la Laguna del Marquesado; 9. M. N. Palancares y Tierra Muerta; 10. M. N. Lagunas de Cañada del Hoyo; 11. R. N. Complejo Lagunar de Arcas; 12. R. N. Laguna de El Hito; 13. Futuro ENP de la Laguna de El Tobar; 14. Futuro ENP Complejo Lagunar de Fuentes; 15. Futuro ENP Rodenal del Cabriel.

Fig. 2.- Location of Protected Natural Areas (ENP) (vertical lines) and future ENP (horizontal lines) in the province of Cuenca. Legend: 1. Natural Monument (N. M.) of "Serrezuela de Valsalobre"; 2. N. M. of "Hoz de Beteta y Sumidero Mata Asnos"; 3. N. M. of "Torcas de Lagunaseca"; 4. Natural Park (N. P.) of "Alto Tajo"; 5, N. M. of "Muela Pinilla y del Puntal"; 6. N. M. of "Nacimiento del río Cuervo"; 7. N. P. of "Serranía de Cuenca"; 8. Natural Reserve (N. R.) of "Laguna del Marquesado"; 9. N. M. of "Palancares y Tierra Muerta"; 10. N. M. of "Lagunas de Cañada del Hoyo"; 11. N. R. of "Complejo Lagunar de Arcas"; 12. N. R. of "Laguna de El Hito"; 13. Future ENP of "Laguna de El Tobar"; 14. Future ENP of "Complejo Lagunar de Fuentes"; 15. Future ENP of "Rodenal del Cabriel".

La figura 1 indica la recopilación de la distribución corológica de Graellsia isabelae en la provincia de Cuenca y limítrofes, observándose una amplia distribución con un total de 30 presencias en cuadrículas UTM 10x10 km en la Serranía de Cuenca y Montes Universales, 19 de dichas cuadrículas pertenecen a la provincia de Cuenca o con compartidas con las provincias de Teruel y Valencia y 8 cuadrículas amplían la distribución conocida según la bibliografía consultada (Aistleitner \& Aistleiner, 1998; Galante \& Verdú, 2000; Montagud et al., 2001; Piñas, 2003) y los comentarios personales de Jordi Domingo y Joaquín Baixeras.

Según el mapa de distribución, las citas más meridionales del Sistema Ibérico son las que corresponden a 30SWK81 y 30SXK51 (límite entre Cuenca y Valencia), aunque la cita más al sur de la Serranía de Cuenca que se ha encontrado en la 
Tabla 3.- Información sobre el cruce de datos entre las UTM de 1x1 km de lado conocidas con las capas GIS: Mapa Forestal, Geología (A15: Rocas básicas del Jurásico, A16: Rocas básicas del Cretácico), Clasificación climática de AlluéAndrade (1990): VI(IV)1, Nemoromediterráneo genuino, variante de áreas altibasales interiores; VI(IV)2, Nemoromediterráneo genuino, variante de media montaña; VIII(VI), Oroarticoide subnemoral y rango altitudinal.

Table 3.- Environmental characterization of the $1 \times 1 \mathrm{~km}$ occurrences of the species: forest map, climatic characterization of Allué-Andrade (1990): VI(IV)1, Nemoromediterranean genuine, variant altibasales interior areas, VI(IV)2, Nemoromediterranean genuine, variant mid-mountain, VIII(VI), Subnemoral oroarticoid, geological preferences (A15: Jurassic basic rocks, A16: Cretaceous basic rocks) and altitude.

\begin{tabular}{lcccc}
\hline ESPECIE/PATRÓN & Vegetación Principal & Preferencias Geológicas & Caracterización Allué-Andrade & Rango altitudinal \\
\hline G. isabelae & Pinus nigra (63\%) & & $\mathrm{VI}(\mathrm{IV}) 1(35 \%)$ & \\
& Pinus sylvestris (21\%) & $\mathrm{A} 15(25 \%)$ & $\mathrm{VI}(\mathrm{IV}) 2(60 \%)$ & $840-1860$ \\
\hline
\end{tabular}

bibliografía sería la de Gómez-Bustillo \& Fernández-Rubio (1976) en la localidad de Motilla del Palancar (30SWJ98). Respecto a esta cita, no hay presencia en el término municipal de ninguna de sus plantas nutricias según el mapa forestal consultado, estando a $2,5 \mathrm{~km}$ la masa más cercana de $P$. nigra subsp. salzmanii. Sin embargo, según Montoya \& Hernández (1975) el imago tendría un fuerte vuelo de dispersión que puede llegar a los 10 $\mathrm{km}$ y podría verse atraído por la contaminación luminosa del núcleo urbano de Motilla del Palancar, tal y como ocurre en otros núcleos de la comarca de la Sierra (Joaquin Baixeras y Jordi Domingo, com. pers.) por lo que la presencia aquí puede ser posible, al menos de modo accidental. Así mismo, otro dato que puede hacer creíble su localización en Motilla del Palancar es que, como se verá más adelante, la cuadrícula 30SWJ98 estaría recogida dentro del mapa potencial de distribución UTM de 10x10.

Presencia en los Espacios Naturales Protegidos Teniendo en cuenta las Figuras 1 y 2, G. isabelae, según su distribución UTM 10x10, estaría presente en los siguientes ENP: Parque Natural de la Serranía de Cuenca, como ya citaron De Arce \& Jiménez (2007); P. N. Alto Tajo; Monumento Natural de Palancares y Tierra Muerta; M.N. Lagunas de Cañada del Hoyo; M. N. Hoz de Beteta y Sumidero Mata Asnos; Reserva Natural de la Laguna del Marquesado; y en el futuro ENP de la Laguna del Tobar, por lo que esta mariposa nocturna se encontraría protegida dentro de los límites de los mencionados ENP, coincidiendo con su inclusión en cada una de las resoluciones de declaración de los ENP citados anteriormente.

Sin embargo, según el mapa de distribución de la Figura 1, no está confirmada la presencia de G. isabelae en los ENP Monumento Natural Muela Pinilla y del Puntal y en el M. N. Serrezuela de Valsalobre, a pesar de ser mencionada en sus correspondientes declaraciones de protección. La presencia de esta mariposa en ambos Monumentos Naturales es muy posible, tal y como se verá más adelante en el mapa potencial UTM 10x10, debido a la abundancia de sus plantas nutricias ( $P$. nigra subsp. salzmanii y $P$. sylvestris) en la zona, pero no existe cita alguna con localidad y fecha que lo corrobore.

CARACTERÍSTICAS AMBIENTALES DEL ÁREA DE PRESENCIA

La Tabla 3 ofrece información sobre la superposición de los datos conocidos de las UTM de 1x1 $\mathrm{km}$ de lado con respecto a las capas GIS: Mapa Forestal, Geología, Clasificación climática de Allué Andrade (1990) y Modelo Digital del Terreno. Para ello, se han utilizado las citas UTM 1x1 km de la Tabla 2 junto con las citas presentes en Piñas (2003): Caserío Embid, una oruga el 20/07/1971 (30TWK7243) y en Galante \& Verdú (2000): C.F. Prado Tejero/ Sierra de los Barrancos (30TWK 8477); Serranía de Cuenca/ Río Caz (30TWK8853); Tragacete/ La Alconera (30TWK9463); Robalbillo/ Tierra Muerta (30TWK 9741); Hoz de Valdemorillo-Vega (30TXK0133); Estación Geodésica Valdemeca/ Valdemoro de la Sierra (30TXK0547); Tobar (30TXK0944); Cañada Rodrigo (Huerta del Marquesado) (30TXK1246). 


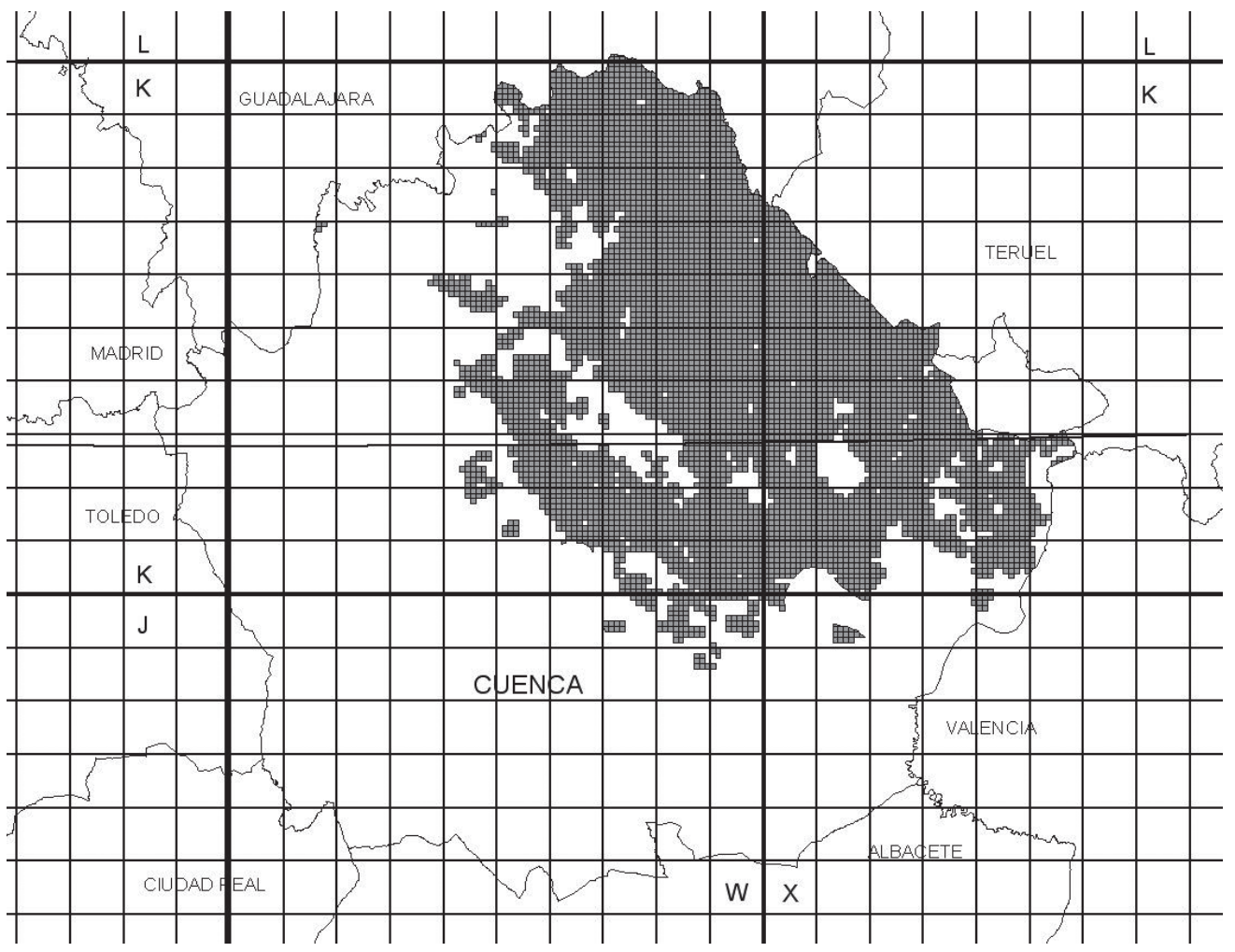

Fig. 3.- Mapa de distribución potencial de Grael/sia isabelae en la provincia de Cuenca sobre cuadrículas UTM 1x1 km.

Fig. 3.- Potential distribution map of Grael/sia isabelae in the province of Cuenca using $1 \times 1 \mathrm{~km}$ UTM squares.

Según la Tabla 3, la vegetación principal dominante son las masas forestales de pino laricio, P. nigra subsp. salzmanii (63\%) y pino albar, Pinus sylvestris $(21 \%)$, que suponen un total del $84 \%$, datos que se asemejan a los proporcionados por Chefaoui \& Lobo (2007) que indican que hay un $82 \%$ de concordancia entre ambos tipos de pinos. Por otro lado, al contrario que en Masó et al. (1989), hay una correlación más alta entre $P$. nigra subsp. salzmanii y la distribución de $G$. isabelae que con P. sylvestris; probablemente la razón es de simple disponibilidad más que de preferencia, debido a la superior extensión ocupada por el pino negral en Cuenca, la provincia española con mayor superficie poblada por esta especie: 142.000 hectáreas de $P$. nigra frente a 30.000 de $P$. sylvestris y 26.000 de ambas en mezcla (MMA, 1996). Como veremos a continuación, el resto de parámetros ecológicos son plenamente concordantes con la unión de los rangos de estos dos árboles.

En cuanto a la localización de $G$. isabelae con respecto a las preferencias geológicas, cabe destacar que está presente sobre terrenos de naturaleza básica en un $62,5 \%$ (rocas del Jurásico y Cretácico), en discordancia con Chefaoui \& Lobo (2007) en el que hay una correlación negativa de sustrato calcáreo y positiva con el sustrato silíceo. Este dato no es significativo, ya que en la provincia de Cuenca tienen una mayor presencia las rocas calcáreas, encontrándose las rocas silíceas en pequeños núcleos. Desde el punto de vista geobotánico, la especie Pinus nigra subsp. salzmanii, si bien en el conjunto de su distribución ibérica puede formar magníficas masas sobre sustrato silíceo, en general abunda más en calizo (Blanco et al., 1997; Ruiz de la Torre, 2006) y en Cuenca se halla principalmente sobre calizas y dolomías e incluso yesos (Martínez Labarga, 2009); sin embargo P. sylvestris es indiferente a la litología de la zona encontrándose también en rocas de naturaleza silícea (rocas Triásicas) como sucede en las Sierras de Valdemeca y Tragacete.

De acuerdo a los datos de altitud disponibles, el rango altitudinal de G. isabelae abarca la totalidad 


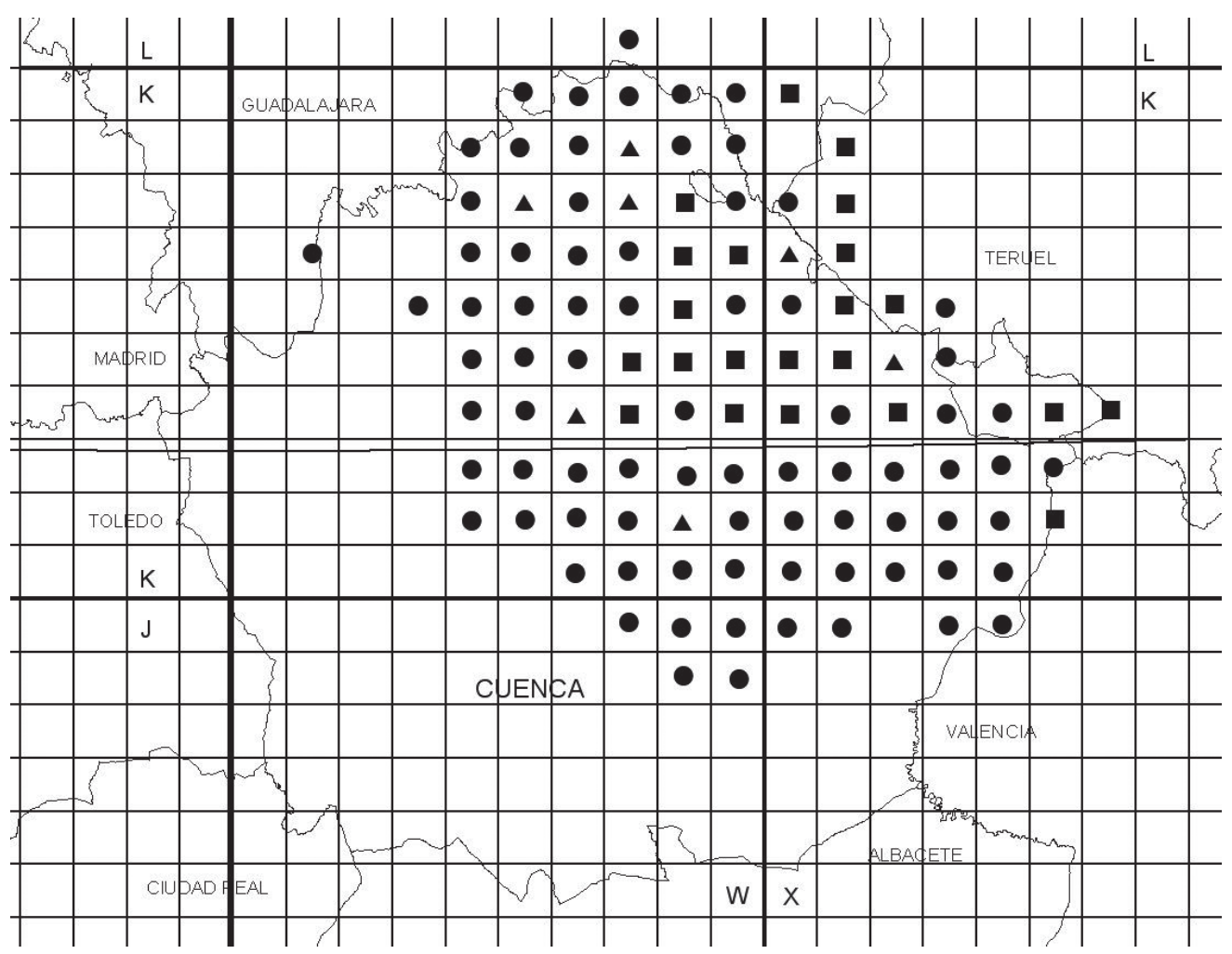

Fig. 4.- Mapa de distribución potencial de Grael/sia isabelae en la provincia de Cuenca sobre cuadrículas UTM 10x10 km. Leyenda: el símbolo cuadrado indica las citas obtenidas de la bibliografía, los triángulos indican las cuadrículas que amplían la distribución y los círculos indican las cuadrículas UTM que amplían distribución con respecto al mapa potencial UTM 1x1.

Fig. 4.- Potential distribution map of Graellsia isabelae in Cuenca using 10x10 km UTM squares. Squares: references from the literature, triangles: new records, circles: UTM squares containing suitable 1x1 UTM squares.

de la Serranía de Cuenca, encontrándose entre los 820 y los $1860 \mathrm{~m}$ de altitud. De nuevo concuerda con sus plantas nutricias, ya que la especie $P$. sylvestris se encuentra preferentemente en altitudes superiores a $1400 \mathrm{~m}$, alcanzando las máximas cotas provinciales en la Mogorrita (1866 m); por su parte la especie $P$. nigra subsp. salzmanii es abundante en el piso medio entre los $1200 \mathrm{y}$ los $1500 \mathrm{~m}$ de altitud, aunque también se puede encontrar por debajo de los $1200 \mathrm{~m}$ en el piso inferior, normalmente en exposiciones de umbría (García Cardo, 2007).

De acuerdo a la clasificación de Allué-Andrade, G. isabelae se extiende por todo el rango del tipo VI(IV), Nemoromediterráneo Genuino y el VIII(VI), Oroarticoide subnemoral. Si bien en éste último pueden ser frecuentes las temperaturas mínimas inferiores a las señaladas por Ylla (1997) como letales para las crisálidas (en el entorno de -12 a $-16^{\circ} \mathrm{C}$ ), la capa- cidad de dispersión de los adultos desde el piso inferior debe permitirle recolonizar con cierta facilidad las máximas cotas provinciales.

\section{DISTRIBUCIÓN POTENCIAL}

En Chefaoui \& Lobo (2007) se identifican como buenas variables predictoras de distribución de $G$. isabelae la presencia de sus plantas nutricias $P$. sylvestris y $P$. nigra subsp. salzmanii, la precipitación estival, la aridez y una altitud media. En el presente trabajo, se presenta la distribución potencial 1x1 $\mathrm{km}$ de lado de G. isabelae (Figura 3) con respecto a la distribución de sus plantas nutricias (mapa forestal de pino laricio y pino albar), las cotas altitudinales (entre 840 y $1860 \mathrm{~m}$ ) y la caracterización climática de Allué-Andrade [VI(IV)1, VI(IV)2, y VII(VI)], sin tener en cuenta las preferencias geológicas. La Figura 4 presenta la distribución bioge- 
ográfica en cuadrículas de 10×10 km de lado en base a la distribución del mapa potencial UTM de cuadrículas de $1 \mathrm{x} 1 \mathrm{~km}$ de la Figura 3.

En la Figura 3 hay un total de 6.010 cuadrículas UTM de 1x1 y en la Figura 4 hay 107 cuadrículas de $10 \times 10$ con presencia de la especie en las provincias de Cuenca, Guadalajara, Teruel y Valencia. Si se utilizan únicamente las variables mapa forestal y curvas de nivel se obtiene un total de 6.066 cuadrículas UTM 1x1, pero al trasladar esta información a un mapa 10x10 se consigue el mismo número de cuadrículas: 107; por lo que no se produce modificación alguna si se trabaja a la escala de 10x10 km de lado.

Según el mapa potencial (Figura 4) podría haber condiciones ambientales favorables para la especie en 77 cuadrículas UTM de $100 \mathrm{~km}^{2}$ de la provincia de Cuenca. Así mismo, la especie podría encontrarse en lugares alejados de la Serranía de Cuenca como la Sierra de Altomira y lugares cercanos como en las Sierras de Bascuñana, Cabrejas, Mira y Aliaguilla entre otras, así como en la parte más oriental de la comarca de la Alcarria de Cuenca.

Con respecto a los ENP y el mapa potencial UTM de $10 \times 10 \mathrm{~km}$ de lado, Figuras 2 y 4 respectivamente, la especie podría estar presente también en el Monumento Natural (M.N.) Serrezuela de Valsalobre, M. N. Torcas de Lagunaseca, M. N. Muela Pinilla y del Puntal, M. N. Nacimiento del río Cuervo, R. N. Complejo Lagunar de Arcas y en los futuros ENP del Rodenal del Cabriel y del Complejo Lagunar de Fuentes, lo que indica que habría que ampliar su cobertura de protección.

Según las Figuras 3 y 4, es de esperar que la prospección de nuevos territorios, poco o nada muestreados hasta la fecha, haga aumentar el número de citas y de cuadrículas UTM con presencia de G. isabelae en Cuenca y en las provincias limítrofes. La colonización de nuevas áreas forestales, debido principalmente a las repoblaciones forestales, es una tendencia que se detecta, como ya apuntaban Gómez-Bustillo \& Fernández-Rubio (1976) y De Viedma \& Gómez Bustillo (1976, 1985), gracias al mayor conocimiento de la entomofauna del territorio estudiado que proporcionan los nuevos estudios.

\section{CONSERVACIÓN}

Según Chefaoui \& Lobo (2007) una de las principales amenazas para la conservación de G. isabelae es la destrucción de su hábitat (incendios forestales, tala, urbanización) y la aplicación de tratamientos químicos contra plagas forestales, sin embargo, ninguno de los dos factores supone un serio problema en esta provincia.

Los pinares albares y laricios son ecosistemas ampliamente representados en el Sistema Ibérico meridional durante todo el Holoceno, dominando el paisaje de modo continuado en las zonas más elevadas o formando mosaicos dinámicos con sabinares y carrascales en las más bajas (Juliá et al., 1998; Stevenson, 2000; Riera, 2006), donde G. isabelae ha encontrado un ambiente óptimo para prosperar. En el último siglo y medio, la gestión forestal ha permitido que la superficie poblada de pinares haya aumentado. Por otro lado, los incendios forestales en la Serranía de Cuenca no representan una amenaza tan seria como en otras regiones. Además, la Serranía es una de las zonas en las que el despoblamiento demográfico es muy grande por lo que la tala de pinos para la urbanización no es un problema para la conservación de la especie.

Los tratamientos contra plagas forestales, en concreto contra la procesionaria del pino (Thaumetopoea pityocampa Denis \& Schiffermüller, 1775), se ha señalado que tienen un impacto negativo sobre la población de $G$. isabelae por el diflubenzurón en emulsión oleosa que tiene una persistencia prolongada sobre el follaje (Soria et al., 1986; Ylla, 1997). Un tratamiento inocuo es la fumigación con formulados de Bacillus thuringiensis var. kurstaki en suspensión acuosa, aplicado en otoño persiste sólo unas dos semanas (De Liñán, 2003), y G. isabelae en esos momentos ya se encuentra en fase de pupa. No obstante, ambos tratamientos han disminuido en los últimos años: el último tratamiento aéreo en Cuenca se realizó en 2004 sobre 4.600 hectáreas con diflubenzurón y 2.800 Ha con Bacillus thuringiensis, principalmente en zonas transitadas (Delegación Provincial de Agricultura, datos inéditos), ya que una infestación elevada crea pérdida de atractivo turístico e incluso alarma social (González, 2008). En los cinco años transcurridos, tanto T. pityocampa como G. isabelae han vuelto a ocupar áreas fumigadas (ej.: Valdecabras, ver Tabla 2). En años posteriores a 2004 se ha cambiado el tratamiento utilizando un control anual en pequeñas superficies mediante trampas de feromona de T. pityocampa. Ambas especies no se excluyen, por lo que resultaría interesante investigar el posible grado de competencia, puesto que presumiblemente las defoliaciones masivas por la procesionaria puedan desplazar local y temporalmente a $G$. isabelae, observándose que los pinos defoliados en general no comienzan a recuperar el 
follaje hasta bien entrada la primavera, pero los imagos de $G$. isabelae en muchos casos han emergido antes y es probable que en su dispersión busquen otras zonas no afectadas por la plaga.

\section{Conclusión}

Grallesia isabelae puede considerarse, en el ámbito de la provincia de Cuenca, una especie en buen estado de conservación, que ocupa un área de distribución relativamente amplia, ligada a un tipo de hábitat extenso y bien conservado, y sin factores preocupantes de amenaza. Su condición de especie protegida se sustenta fundamentalmente en su carácter endémico, emblemático y representativo de algunos de los paisajes forestales más característicos de la región.

\section{Agradecimientos}

Este estudio ha sido financiado en parte por diferentes proyectos del Servicio de Medio Natural de la Delegación de Medio Ambiente de Cuenca (Junta de Comunidades de Castilla- La Mancha). Se agradece la colaboración de Nicolás Hernández y Sandra Ramírez-Cárdenas Amer por comunicarnos una cita, la colaboración desinteresada de Armando DíazRomeral por la consulta de los datos de su colección personal, al Dr. Marcelino de los Mozos Pascual (Centro de Investigación Agraria de Albaladejito-Cuenca), al Dr. Jordi Domingo Calabuig y al Dr. Joaquin Baixeras (Institut Cavanilles de Biodiversitat i Biologia Evolutiva- Universitat de València) por aportar citas al presente trabajo, al Dr. Antonio S. Ortiz Cervantes (Facultad de VeterinariaUniversidad de Murcia) por informar y facilitar bibliografía, a Jesús Madero y Fernando Escaso del Museo de las Ciencias de Castilla- La Mancha (MCCM) por la consulta y revisión de la colección de Francisco Piñas Amor. Por último, agradecer al Dr. Alberto Jiménez Valverde la revisión del texto completo que sin su ayuda no hubiera sido posible su elaboración.

\section{Referencias}

Aistleitner, U. \& Aistleitner, E., 1998., Faunistic records of Bombyces and Sphinges (sensu classico) from Spain (Insecta: Lepidoptera). Shilap Revista de Lepidopterología, 126(103): 147-163.

Allué-Andrade, J. L., 1990. Mapa de Subregiones Fitoclimáticas de España Peninsular y Balear. Formato digital, Ministerio de Medio Ambiente y Medio Rural y Marino: http://www.mma.es/portal/ secciones/biodiversidad/banco_datos/info_disponible/mapa_subregiones_fitoclim. $\bar{h}$ tm
Blanco Castro, E., Casado González, M. A., Costa tenorio, M., Escribano Bombín, R., García Antón, M., Génova Fuster, M., Gómez Manzaneque, F., Moreno Sáiz, J. C., Morla Juriasti, C., Regato Pajares, P. \& Sáinz Ollero, H., 1997. Los Bosques Ibéricos, una interpretación geobotánica. Planeta. Barcelona. 572 pp.

Blázquez, A., Hernández-Roldán, J., Nieto, M. A. \& Garcia-Santano, A., 2000. Nuevos datos sobre la fauna de macroheteróceros de la provincia de Cáceres (España) III (Insecta: Lepidoptera). Shilap Revista de Lepidopterología, 28(110): 173-186.

Calle, J. A. de la, Lencina, F., González, F. \& Ortiz, A. S., 2000. Las mariposas de la Región de Murcia. Macrolepidópteros diurnos y nocturnos. Nausícaä Edición Electrónica / Caja de Ahorros del Mediterráneo. Murcia. 396 pp.

Chefaoui, R. M. \& Lobo, J. M., 2007. Assessing the conservation status of an Iberian moth using pseudoabsences. Journal of Wildlife Management, 71(8): 2507-2516.

De Arce, J. I. \& Jiménez Mendoza, S., 2007. Las mariposas del Parque Natural de la Serranía de Cuenca. Revista Medio Ambiente de Castilla-La Mancha, 14: 18-23.

De Liñán, C., 2003. Farmacología Vegetal, $3^{\text {a }}$ edición. Ediciones Agrotécnicas. Madrid. 1270 pp.

De Viedma, M. G. \& Gómez-Bustillo, M. R., 1976. Libro Rojo de los Lepidópteros ibéricos. Ministerio de Agricultura, Pesca y Alimentación, ICONA. Madrid. $117 \mathrm{pp}$.

De Viedma, M. G. \& Gómez-Bustillo, M. R., 1985. Revisión del Libro Rojo de los Lepidópteros ibéricos. Ministerio de Agricultura, Pesca y Alimentación, ICONA. Madrid. $71 \mathrm{pp}$.

Fernández Irurita, J. M., 2008. Libro Rojo de los Invertebrados de Andalucía. Tomo III: Lepidópteros. Consejería de Medio Ambiente. Versión digital: http://www.juntadeandalucia.es/medioambiente/web /Bloques Tematicos/Publicaciones Divulgacion Y Noticias/Documentos Tecnicos/libro rojo invertebrados/tomo3/orden_lepidoptera.pdf

Galante, E. \& Verdú, J. R., 2000. Los Artrópodos de la "Directiva Hábitat" en España. Colección técnica. Ministerio de Medio Ambiente. Madrid. 247 pp.

García Cardo, O., 2007. La vegetación de la Serranía de Cuenca. Revista Medio Ambiente de Castilla-La Mancha, 14: 14-17.

García-Barros, E. \& Herranz, J., 2001. Nuevas localidades de Proserpinus proserpina (Pallas, 1772) y Graellsia isabelae (Graells, 1849). Shilap Revista de Lepidopterología, 29(114): 183-184.

García-Barros, E., Munguira, M. L., Martín Cano, J., Romo Benito, H., García Pereira, P. \& Maravalhas, E. S., 2004. Atlas de las Mariposas diurnas de la Peninsula Iberica e Islas Baleares (Lep.: Papilionoidea \& Hesperioidea). Sociedad Entomológica Aragonesa (SEA). Zaragoza. 228 pp. 
Gómez-Bustillo, M. R. \& Fernández-Rubio, F., 1974. Mariposas de la Península Ibérica. Ropalóceros I. ICONA Ministerio de Agricultura, Madrid. 198 pp.

Gómez-Bustillo, M. R. \& Fernández-Rubio, F., 1976. Las mariposas de la Península Ibérica. Heteróceros $I$. ICONA. Madrid. 300 pp.

González, E., 2008. La procesionaria del pino (Thaumetopoea pityocampa Den. \& Shiff.): Manual para la evaluación de los niveles de infestación en rodales de seguimiento en Castilla-La Mancha. Junta de Comunidades de Castilla-La Mancha. Consejería de Medio Ambiente y Desarrollo Rural. Serie Técnica: La salud de los bosques, $n^{\circ} 2$.

Guyot, H. (coord.). 1998. Contribution à la connaisance de Graellsia isabelae galliaegloria Oberthür (Lepidoptera, Attacidae) connu uniquement en France. Rapport d'études de l'OPIE. Vol 3. Guyancourt. 36 pp.

Ibáñez Gázquez, S., Nevado Ariza, J. C. \& Ylla Ullastre, J., 2008. Graellsia isabelae (Graells, 1849), una nueva especie para la fauna lepidopterológica de Almería (España) (Lepidoptera: Saturniidae). Shilap Revista de Lepidopterología, 36(144): 427-430.

Jiménez-Valverde, A., Gómez, J. F., Lobo, J. M., Baselga, A. \& Hortal, J., 2008. Challenging species distribution models: the case of Maculinea nausithous in the Iberian Peninsula. Annales Zoologici Fennici, 45: 200-210.

Julià, R., Burjachs, F., Dasí, M. J., Mezquita, F., Miracle, M. R., Roca, J. R., Seret, G. \& Vicente, E., 1998. Meromixis origin and recent trophic evolution in the Spanish mountain lake La Cruz. Aquatic Sciences, 60: 279-299.

Koschwitz, J., Kraus, W. \& Blum, E., 1985. Contribution to the butterfly-fauna of Albarracín (Prov. Teruel) and adyacent areas (I). Shilap Revista de Lepidopterología, 13(50): 125-130.

López-Sebastián, E., López, J. C. \& Selfa, J., 2001. Nota preliminar sobre la distribución de Graellsia isabellae (Graells, 1849) (Lepidoptera, Saturniidae) en la Provincia de Valencia. Graellsia, 57(2): 183-184.

Martínez Labarga, J. M., 2009. Pinus nigra Arnold subsp. salzmannii (Dunal) Franco, sobre yesos en la Alcarria de Cuenca. V Congreso Forestal Español, Ávila: 9 pp.

Masó i Planas, A. \& Ylla Ullastre, J., 1989. Consideraciones sobre la ecología, comportamiento, alimentación y biogeografía de Graellsia isabelae (Graells) (Lep. Saturniidae). Shilap Revista de Lepidopterología, 17(65): 45-60.

Ministerio de Medio Ambiente. 1996. Segundo Inventario Forestal Nacional. Cuenca. Dirección General de Conservación de la Naturaleza. Madrid. 237 pp.

Montagud Alario, S., Domingo Calabuig, J. \& Baixeras Almela, J., 2001. Metodología para el cartografiado de las poblaciones de Graellsia isabelae (Lepidoptera,
Saturniidae): una propuesta aplicada a su conservación en la Comunidad Valenciana. Libro de resúmenes de las XIX Jornadas de la Asociación Española de Entomología, Barcarrota (Badajoz): 85.

Montoya, R. \& Hernández, R., 1975. Graellsia isabelae (Graells). Vida Silvestre, 12: 207-211.

Pérez De Gregorio, J. J., Muñoz, J. \& Rondós, M., 2002. Atlas fotográfico de los lepidópteros macroheteróceros iberobaleares 2. Argania. Barcelona. 214 pp.

Piñas, F., 2003. Mariposas Ropalóceros, en los alrededores de Cuenca. Diputación de Cuenca, Área de Cultura. Cuenca. 185 pp.

Riera, S., 2006. Cambios vegetales holocenos en la región mediterránea de la Península Ibérica: ensayo de síntesis. Revista Ecosistemas, 15(1): 17-30. http: //www.revistaecosistemas.net/articulo.asp? Id=401

Romo, H., García-Barros, E \& Munguira, M. L., 2006. Distribución potencial de trece especies de mariposas diurnas amenazadas o raras en el área ibero-balear (Lepidoptera: Papilionoidea \& Hesperioidea). Boletín de la Asociación Española de Entomología, 30(3-4): 25-49

Ruiz de la Torre, J., 2006. Flora Mayor. Organismo Autónomo de Parques Nacionales. Madrid. 1756 pp.

Soria, S., Abós, F., \& Martín, E., 1986. Influencia de los tratamientos con diflubenzurón ODC 45\% sobre pinares en las poblaciones de Graellsia isabelae y reseña de su biología. Boletín de Sanidad Vegetal. Plagas, 12: 29-50.

Stevenson, A. C., 2000. The Holocene forest history of the Montes Universales, Teruel, Spain. The Holocene, 10: 603-610.

Verdú, J.R. \& Galante, E. (eds.). 2006. Libro Rojo de los Invertebrados de España. Dirección General de Conservación de la Naturaleza, Madrid (versión online). http://carn.ua.es/CIBIO/es/Irie/Irie.html

Viejo, J. L., Martín, J., García-Barros, E. \& Munguira, M.L., 1991. The Bern Convention and the Spanish Lepidoptera. In: The Conservation of Insects and their Habitats, 15 th Symposium RES. N. M. Collins \& J.A. Thomas (eds.). Academic Press. London: 428-429.

Ylla J., 1997. Historia natual del lepidopter Graellsia isabelae (Graells, 1849). Instiyut d'Estudis Catalans. Barcelona. 232 pp. 